\title{
Criteria for Hyponatremic Overcorrection: Systematic Review and Cohort Study of Emergently III Patients
}

\author{
Jason D. Woodfine, $M D^{1,2}$ and Carl van Walraven, $\mathrm{MD}^{1,2,3,4}$
}

'Department of Medicine, The Ottawa Hospital, ASB1-003, Ottawa, Ontario, Canada; ${ }^{2}$ Ottawa Hospital Research Institute, Ottawa, Canada; ${ }^{3}$ Department of Epidemiology \& Community Medicine, The University of Ottawa, Ottawa, Canada; ${ }^{4}$ Institute for Clinical Evaluative Sciences @ uOttawa, Ottawa, Canada.

BACKGROUND: Hyponatremia is the most common electrolyte disturbance amongst hospitalized patients. An overly rapid rate of correction of chronic hyponatremia is believed to increase the risk of poor clinical outcomes including osmotic demyelination syndrome (ODS). There is disagreement in the literature regarding the definition of hyponatremic overcorrection.

METHODS: We performed a systematic review of all English language studies to identify those that calculated sodium correction rate and classified patients' overcorrection status. We then identified all patients who presented to our hospital's emergency department between 2003 and 2015 with a corrected serum sodium $\leq$ $116 \mathrm{mmol} / \mathrm{L}$. All methods from the systematic review for sodium correction rate calculation and overcorrection status were applied to this cohort.

RESULTS: We identified 24 studies citing 9 distinct sodium correction rate methods and 14 criteria for overcorrection. Six hundred twenty-four patients presenting with severe hyponatremia (median initial value $113 \mathrm{mMol}$ ) were identified. Depending on the method used, the median sodium correction rates in our cohort ranged from 0.271 to $1.13 \mathrm{mmol} / \mathrm{L}$ per hour. The proportion of patients classified with overcorrection with the different criteria varied almost 11 -fold, ranging from 8.5 to $89.9 \%$.

CONCLUSION: Published methods disagree regarding the calculation of sodium correction rates and the definition of hyponatremic overcorrection. This leads to wide variations in sodium correction rates and the prevalence of overcorrection in patient cohorts. Definitions based on ODS risk are needed.

\footnotetext{
Clinical Significance

- This is the most extensive examination of methods used to calculate hyponatremia correction rates and definitions of overcorrection.

- There is little evidence available to guide definitions of overcorrection.

- This is the first study to directly compare the different definitions of overcorrection in a large cohort study.

- The heterogeneity of overcorrection definitions directly influences the prevalence of overcorrection in a given cohort
}

Electronic supplementary material The online version of this article (https://doi.org/10.1007/s11606-019-05286-y) contains supplementary material, which is available to authorized users.

Received October 18, 2018

Revised April 3, 2019

Accepted July 24, 2019

Published online August 26, 2019
KEY WORDS: hyponatremia; osmotic demyelination syndrome; outcome measurement; methodology.

J Gen Intern Med 35(1):315-21

DOI: $10.1007 / \mathrm{s} 11606-019-05286-\mathrm{y}$

(c) Society of General Internal Medicine 2019

\section{INTRODUCTION}

Hyponatremia is the most common electrolyte disorder encountered in the hospital, affecting more than 1 in 4 patients. ${ }^{1}$ Hyponatremia presents clinically from mild changes in cognition to potentially fatal comas that necessitate emergent treatment. The gravest outcome of hyponatremic treatment is osmotic demyelination syndrome (ODS) or central pontine myelinolysis. Studies have identified certain non-modifiable patient characteristics including a profoundly low initial serum sodium, cirrhosis, malnutrition, and hypokalemia that increase the risk of ODS when hyponatremia is corrected. ${ }^{2,3}$ The most recognized modifiable risk factor for ODS is the sodium correction rate. ${ }^{4}$ Since physicians can influence the sodium correction rate, clinicians usually commit a great deal of energy and concern to avoid hyponatremic overcorrection.

However, the definition of hyponatremic overcorrection is unclear. Such a definition must describe both a method by which the sodium correction rate is calculated and an overcorrection threshold (i.e., a value that categorizes the former into the presence or absence of overcorrection). For example, American guidelines ${ }^{4}$ recommend that sodium correction rates are calculated by simply determining the change in serum sodium over the specified period to return an average rate of sodium change; overcorrection is present if this rate exceeds $10-12 \mathrm{mMol} / \mathrm{L}$ per $24 \mathrm{~h}$ or $18 \mathrm{mMol} / \mathrm{L}$ per $48 \mathrm{~h}$ with an even more stringent target of $8 \mathrm{mMol} / \mathrm{L}$ per $24 \mathrm{~h}$ for those patients at highest risk of developing osmotic demyelination syndrome. However, European guidelines ${ }^{5}$ define overcorrection as a rate exceeding $10 \mathrm{mmol} / \mathrm{L}$ during the first $24 \mathrm{~h}$ or $8 \mathrm{mMol} / \mathrm{L}$ in any 24-h period thereafter, but do not explicitly state the method by which the sodium correction rate is calculated. Both sets of guidelines acknowledge the limited evidence behind these recommendations.

The distinctions in the American ${ }^{4}$ and European ${ }^{5}$ guidelines alone indicate that variation exists in the definition of acceptable hyponatremia correction rates. However, the extent of this 
Table 1 Measurement of Serum Sodium Correction Rates and Threshold for Overcorrection

\begin{tabular}{|c|c|c|c|c|c|c|c|}
\hline \multicolumn{2}{|c|}{ Sodium correction rate } & \multicolumn{6}{|c|}{ Overcorrection threshold } \\
\hline & & \multirow{3}{*}{$\begin{array}{l}1 \\
0.5 \frac{\mathrm{mmol}}{\mathrm{L}} \text { per hour } \\
10,11 *\end{array}$} & \multirow{2}{*}{$\frac{2}{8 \frac{\mathrm{mmol}}{\mathrm{L}} \text { per } 8 h}$} & \multirow{2}{*}{$\begin{array}{l}3 \\
8 \frac{\mathrm{mmol}}{\mathrm{L}} \text { per day }\end{array}$} & \multirow{2}{*}{4} & \multirow{2}{*}{$\begin{array}{l}5 \\
12 \frac{\mathrm{mmol}}{\mathrm{L}} \text { per day }\end{array}$} & \multirow{2}{*}{$\begin{array}{l}\mathbf{6} \\
18 \frac{\mathrm{mmol}}{\mathrm{L}} \text { per } 2 \text { days }\end{array}$} \\
\hline Name & & & & & & & \\
\hline & $\frac{N a_{\text {final }}-\mathrm{Na}_{\text {intitial }}}{\text { hours }}$ & & & & & & \\
\hline B & $\frac{\mathrm{Na}_{\mathrm{t}=I \mathrm{day}}-\mathrm{Na}_{\mathrm{t}=0}}{l \text { day }}$ & & & 12,13 & & $10,12-19$ & \\
\hline $\mathrm{C}$ & $\frac{\mathrm{Na}_{\mathrm{t}=2 \text { days }}-\mathrm{Na}_{\mathrm{t}=0}}{2 \text { days }}$ & & & & & & $14-17$ \\
\hline $\mathrm{D}$ & $\frac{\mathrm{Na}_{\operatorname{maximum}}-\mathrm{Na}_{\mathrm{t}}=0}{8 \mathrm{hours}}$ & & 20,21 & & & & \\
\hline $\mathrm{E}$ & $\frac{\mathrm{Na}_{\operatorname{maximum}}-\mathrm{Na}_{\mathrm{t}=0}}{l \text { day }}$ & & & 22,23 & $24-26$ & $20,21,27-31$ & \\
\hline $\mathrm{F}$ & $\frac{\mathrm{Na}_{\operatorname{maximum}}-\mathrm{Na}_{\mathrm{t}=0}}{2 \text { days }}$ & & & & & & $27-31$ \\
\hline G & $\frac{\mathrm{Na}_{\text {estimated }}-\mathrm{Na}_{\mathrm{t}=0}}{I \text { day }} *$ & & & 22 & 22,32 & & \\
\hline $\mathrm{H}$ & $\frac{\mathrm{Na}_{\text {estimated }}-\mathrm{Na}_{\mathrm{t}}=0}{2 \text { days }} *$ & & & & & & 22,32 \\
\hline I & $\begin{array}{l}\text { Average daily area } \\
\text { under the Na vs. } \\
\text { Time curve }\end{array}$ & & & & & 33,34 & 34 \\
\hline
\end{tabular}

$\epsilon_{N a_{t=0} \text { represents the first serum sodium measurement }}$

${ }^{{ }_{N}} N a_{t=X}$ represents the serum sodium measurement closest in proximity to time $X$

${ }^{¥} N a_{\text {maximum }}$ represents the largest serum sodium measurement during the observation period

${ }^{\mu} N a_{\text {estimated }}$ represents the time-weighted estimate of the serum sodium measurement at a particular timepoint (specified in the denominator). This method imputed the Na at the timepoint using linear interpolation between Na values prior to and following the timepoint (George et al. CJASN 2018). Similar methods were used if Na was measured only once within $12 \mathrm{~h}$ of the timepoint, in which case linear interpolation was calculated using the baseline Na. This rate was undefined for patients without Na measures within $12 \mathrm{~h}$ of the timepoint

Rates will be undefined for D, E, and F if patients did not have a Na measured between the initial measure and the timepoint indicated in the denominator of the equation

* Reference ${ }^{10}$ calculated this rate using any sequential sodium measures throughout the first $72 \mathrm{~h}$ of the admission (such that $N a_{\text {initial }}$ was the $n^{\text {th }}$ sodium and $N a_{\text {final }}$ was the $(n+1)^{\text {th }}$ sodium). Reference ${ }^{11}$ used this formula (with $N a_{\text {initial }}$ being the sodium prior to the correction intervention and $N a_{\text {final }}$ being the sodium at the end of correction) but did not apply any overcorrection threshold

variation and its influence on the prevalence of hyponatremic overcorrection is unknown. In this study, we conducted a systematic review of the English literature to identify all studies that calculated sodium correction rates and provided a definition of overcorrection. We then applied each of these criteria to a cohort of patients with severe hyponatremia seen in our hospital's emergency department to measure the agreement between the correction rates and overcorrection status from these studies.

\section{METHODS}

\section{Systematic Review}

After consulting a library information specialist, we developed a search strategy for the MEDLINE, EMBASE, and Cochrane Central Register of Controlled Trials databases (Appendix A). JDW reviewed each citation's abstract to eliminate those which clearly did not meet our final inclusion criteria. We reviewed the full text of the remaining citations to identify those meeting our final inclusion criteria which included (a) cohort or randomized controlled study design; (b) explicit definition of sodium correction rate and criteria for sodium overcorrection; (c) inclusion of at least 10 patients; and (d) published in English since 1975. We used these criteria because a cohort design was required to measure changes in sodium concentration over time; explicit definitions of correction rates and criteria were required to apply them to our cohort; the study sample needed to be large enough for us to compare our results; and we found very few studies meeting our criteria prior to 1985 , so we reasoned that limiting our search to post 1975 had very little risk of missing important papers. We did not publish a protocol for this study.

From each included study, we used a spreadsheet package to abstract the methods used to calculate the sodium correction rate. We also identified thresholds for sodium correction rates which defined overcorrection and reasons why these methods or criteria were used (if provided). If a range was used for the overcorrection threshold, the upper limit of the range was recorded.

\section{Cohort Study}

This study took place at a 1000-bed teaching hospital that is the primary referral center in our region of Ontario, Canada. Publicly funded health insurance covered all fees for physician activities, investigations, emergency department visits, and hospitalizations. ${ }^{6}$ 
Table 2 Description of 624 Emergency Department Patients with Severe Hyponatremia

\begin{tabular}{ll}
\hline \hline Factor & Value \\
\hline Mean patient age (SD) & $68.2 \pm 15.5$ \\
Female & $379(60.7 \%)$ \\
Median initial Na (IQR) & $113(110-114)$ \\
Malnourished* & $40(6.4 \%)$ \\
Serum K $<3.0 \mathrm{mMol}$ & $144(23.1 \%)$ \\
eGFR $<30 \mathrm{~mL} / \mathrm{min} / 1.73 \mathrm{~m}^{2}$ & $80(12.8 \%)$ \\
Admitted to hospital & $616(98.7 \%)$ \\
Died in ED or hospital & $59(9.5 \%)$ \\
Median encounter duration in days (IQR) & $7(3-13)$ \\
\hline *Stated in any consult note. SD, standard deviation; $I Q R$, interquartile \\
range; K, potassium; eGFR, estimated glomerular filtration rate; ED, \\
emergency department
\end{tabular}

We used our hospital's admission registry to identify all patients who presented to our hospital's emergency department between 1 January 2003. These dates correspond to the time when data were first complete and long-term follow-up was available. Of these patients, we identified the initial serum sodium measurement during their emergency department encounter and used concurrent serum glucose measures to calculate the corrected serum sodium using a modification of the correction equation from Katz. ${ }^{7}$

Corrected $(\mathrm{Na})=\mathrm{Na}+2 * \frac{\text { Glucose }(\mathrm{mMol})-5.5}{5.5}$

We increased the coefficient in Katz's equation from 1.6 to 2 to ensure that patients included in our study truly had hypotonic hyponatremia. Patients without a serum glucose measure were imputed a normal value. Patients were included in our study if their initial corrected serum sodium was less than $116 \mathrm{mMol} / \mathrm{L}$ and had their sodium measurement repeated at least once during their encounter, either in the emergency department or the subsequent hospitalization. We chose this threshold of $116 \mathrm{mMol} / \mathrm{L}$ because we were primarily interested in studying ODS in our cohort and this outcome is exceedingly rare in patients whose sodium concentrations are above this threshold. We included only the patient's first encounter with our hospital for severe hyponatremia. We identified all serum sodium measurements on each patient during their emergency department and hospital encounter.

\section{Analysis}

We applied all methods identified in our systematic review for calculating sodium correction rate and defining overcorrection to each patient in our cohort study. Rates were standardized to change in $\mathrm{mmol} / \mathrm{L}$ per hour to permit comparisons between rates. We used the Pearson correlation coefficient to calculate correlation between sodium correction rates. Mean absolute differences with $95 \%$ confidence intervals were used to quantify agreement between rates. Because the prevalence of overcorrection was less than $5 \%$ for some methods, we used the prevalence-adjusted bias-adjusted kappa (PABAK) statistic to measure agreement between methods for classifying overcorrection. ${ }^{8}$

\section{RESULTS}

\section{Systematic Review}

Our search strategy (Appendix A) returned 495 citations. Manual review of these citations revealed 84 that were potentially relevant. Twenty-four of these studies met our inclusion criteria (Appendix B) ${ }^{10,22,12-34}$. Most studies evaluated patients in the emergency department or hospital setting $(n=20)$. A justification for the chosen overcorrection criteria was provided in all 24 $(100 \%)$ studies; however, these justifications relied predominantly on information provided in textbooks or guidelines. A single study justified their overcorrection criteria with a citation of an article which found an association between ODS and change in serum sodium concentration of more than $25 \mathrm{mmol} / \mathrm{L}$ in the first $48 \mathrm{~h}$. Oddly, this study found no association between this correction rate and ODS risk. ${ }^{9}$

Within these studies, we identified 9 sodium correction rate formulae and 14 sodium correction rate-overcorrection thresholds combinations (Table 1). Sodium correction rate formulae ranged from simply dividing the difference between each sodium and its subsequent value by the number of hours between the measures (Table 1, rate A) to calculate the area under the Na vs. time curve (Table 1, rate I). The observation periods chosen for calculating rates included $8 \mathrm{~h}$ (rate D),

Table 3 Comparison of Sodium Correction Rates in 624 Patients

\begin{tabular}{|c|c|c|c|c|c|c|c|c|}
\hline \multirow[t]{2}{*}{ Sodium correction rate } & \multirow{2}{*}{$\begin{array}{l}\text { Patients with } \\
\text { incalculable rates }\end{array}$} & \multicolumn{7}{|c|}{ Rate (mmol/h) } \\
\hline & & Minimum & 5th \% ile & 25th \%ile & Median & 75th \% ile & 95th \% ile & Maximum \\
\hline A* & $0(0 \%)$ & -2.286 & 0.398 & 0.741 & 1.131 & 1.907 & 6.000 & 360 \\
\hline B & $0(0 \%)$ & -0.167 & 0.042 & 0.208 & 0.333 & 0.50 & 0.792 & 2.250 \\
\hline $\mathrm{C}$ & $0(0 \%)$ & -0.083 & 0.063 & 0.188 & 0.271 & 0.354 & 0.479 & 1.062 \\
\hline $\mathrm{D}$ & $104(16.7 \%)$ & -0.500 & -0.125 & 0.125 & 0.375 & 0.625 & 1.250 & 4.000 \\
\hline $\mathrm{E}$ & $2(0.3 \%)$ & -0.167 & 0.083 & 0.208 & 0.333 & 0.458 & 0.792 & 2.250 \\
\hline $\mathrm{F}$ & $0(0 \%)$ & -0.083 & 0.083 & 0.188 & 0.271 & 0.354 & 0.479 & 1.208 \\
\hline $\mathrm{G}$ & $11(1.8 \%)$ & -0.080 & 0.053 & 0.228 & 0.360 & 0.510 & 0.819 & 2.281 \\
\hline $\mathrm{H}$ & $71(11.3 \%)$ & -0.185 & 0.131 & 0.379 & 0.576 & 0.714 & 0.960 & 2.127 \\
\hline I1 & $0(0 \%)$ & -0.167 & 0.051 & 0.225 & 0.354 & 0.503 & 0.798 & 2.281 \\
\hline $\mathrm{I} 2$ & $0(0 \%)$ & -0.092 & 0.063 & 0.188 & 0.274 & 0.354 & 0.475 & 1.064 \\
\hline
\end{tabular}

Values are change in mmol serum sodium per hour of observation. Please see Table 1 for definition of how each rate was calculated

*The maximal sodium correction rate calculated during the initial $72 \mathrm{~h}$ of the admission is reported 
1 day (rates B, E, G, I), 2 days (rates $\mathrm{C}, \mathrm{F}, \mathrm{H}, \mathrm{I}$ ), or the time period between two sequential sodium measures (rate A). The final sodium value selected for the calculation varied from the maximum value during the observation period (rates D, E, F) or the value at the end of the observation period, which was defined either as that closest to the observation end (rates A, B, C) or an imputed value using linear interpolation (rates $\mathrm{G}, \mathrm{H}$ ). Significant variations in overcorrection thresholds were also identified, varying from as low as $8 \mathrm{mmol} / \mathrm{L}$ per day (i.e., $0.333 \mathrm{mmol} / \mathrm{L}$ per hour) to $8 \mathrm{mmol} / \mathrm{L}$ per $8 \mathrm{~h}$ (i.e., $1 \mathrm{mmol} / \mathrm{L}$ per hour).

\section{Cohort Study}

We identified 624 patients who were seen in the emergency department between 1 March 2003 and 12 December 2015 with an initial corrected serum sodium value less than $116 \mathrm{mmol} / \mathrm{L}$ (Table 2). These patients were older (mean age 68 years) and mostly female. Hyponatremia was very severe with a median initial serum sodium of $113 \mathrm{mmol} / \mathrm{L}$ (IQR 110 114 ) and almost a quarter of patients having a serum potassium less than $3 \mathrm{mmol} / \mathrm{L}$. Renal dysfunction (eGFR $<30 \mathrm{~mL} /$ min) was noted in $12.8 \%$ of patients. Almost all patients were admitted to hospital. These hyponatremic patients had an incidence of death in hospital of $9.5 \%$, notably exceeding the overall crude in-patient death risk during this time of $3.4 \%$.

When we applied the sodium correction rates identified in our systematic review (Table 1) to our study cohort, notably different rates resulted (Table 3). Four methods were incalculable for some patients, with rate $\mathrm{D}$ being so in one-sixth of patients, which was due to the sodium not being repeated within $8 \mathrm{~h}$ of the initial value in 104 (16.7\%) patients. The median sodium correction rates varied extensively from $0.131 \mathrm{mmol} / \mathrm{L}$ per hour (rate A) to $1.131 \mathrm{mmol} / \mathrm{L}$ per hour (rate A). Using rate A, one patient had an extraordinarily high outcome of $360 \mathrm{mmol} / \mathrm{h}$ when their sodium increased from 125 to $131 \mathrm{mmol} / \mathrm{L}$ when measured $1 \mathrm{~min}$ apart. As a result, the range within specific correction rates also varied tremendously from $1.145 \mathrm{mmol} / \mathrm{L}$ per hour (rate C) to $359 \mathrm{mmol} / \mathrm{L}$ per hour (rate D).

\section{Agreement Between Criteria}

The correlations and differences between the sodium correction rates indicated that several of the rate calculation methods produced results that were notably distinct from the others (Table 4). Rate A had the largest values and lowest correlations with the other rate calculation methods (mean Pearson correlation coefficient 0.065 ), although they remained statistically distinct from 0 with rates $\mathrm{E}$ and $\mathrm{F}$. Values calculated with rate $\mathrm{H}$ also differed extensively in values compared with the other methods. Several methods returned rates that were almost identical, such as rates B and E. Some rates were strongly correlated but returned large differences, such as rates $\mathrm{H}$ and $\mathrm{I}$.

The proportion of patients classified as overcorrected also varied extensively between the different criteria (Table 5). 
Table 5 Prevalence of Overcorrection by Different Criteria

\begin{tabular}{llll}
\hline \hline Rate & $\boldsymbol{N}$ & Overcorrected & \% (95\% CI) \\
\hline A1 & 624 & 561 & $89.9(87.5,92.3)$ \\
B3 & 624 & 310 & $49.7(45.7,53.6)$ \\
B5 & 624 & 152 & $24.4(21,27.7)$ \\
C6 & 624 & 102 & $16.3(13.4,19.3)$ \\
D2 & 520 & 44 & $8.5(6.1,10.9)$ \\
E3 & 622 & 292 & $46.9(43,50.9)$ \\
E4 & 622 & 193 & $31(27.4,34.7)$ \\
E5 & 622 & 125 & $20.1(16.9,23.3)$ \\
F6 & 624 & 109 & $17.5(14.5,20.5)$ \\
G3 & 613 & 326 & $53.2(49.2,57.1)$ \\
G4 & 613 & 245 & $40(36.1,43.9)$ \\
H6 & 552 & 110 & $19.9(16.6,23.3)$ \\
I5 & 624 & 159 & $25.5(22.1,28.9)$ \\
I6 & 624 & 111 & $17.8(14.8,20.8)$ \\
\hline
\end{tabular}

The criterion used to define overcorrection is based on a sodium correction rate method (letter) and overcorrection threshold (number) defined in Table 1

Overcorrection prevalence ranged almost 11 -fold, from a minimum of $8.5 \%$ of patients (criterion D2) to more than almost $90 \%$ of all patients $(89.9 \%$, criterion A1) with a median proportion of $24.9 \%$. Fifty-nine patients $(9.5 \%)$ met no criteria for overcorrection; excluding the strictest criterion A1, 268 patients $(43.0 \%)$ met no criteria. Twenty patients $(3.2 \%)$ met all 14 criteria. Excluding patients who met none of the overcorrection criteria, the distribution for the proportion of criteria for overcorrection was uniform. Agreement for classifying overcorrection also varied widely between the criteria (Table 6). Criteria that used the same rate correction equation but different thresholds (e.g., criteria B3 and B5, criteria G3 and G4) tended to have greater agreement. Criteria A1 had the least agreement with other criteria (mean PABAK $=-0.25$ ) while criteria $\mathrm{B} 5$ and $\mathrm{I} 5$ had the highest agreement with the other criteria (mean PABAKs of 0.56 and 0.57 , respectively).

\section{DISCUSSION}

To our knowledge, this is the most extensive examination of methods used to calculate hyponatremia correction rates and defining hyponatremic overcorrection. Our systemic review of the English literature identified 14 distinct methodologies used to determine hyponatremic overcorrection. When these criteria were applied to over six hundred patients presenting to our emergency department with severe hyponatremia, we found large variations in, and disagreements between, sodium correction rates. Depending on the criterion used, prevalence of overcorrection varied more than 12 -fold.

Our study has several important findings. First, there are few data available to guide definitions of overcorrection. Of the articles identified in our systematic review, only one cited actual data measuring the association between sodium correction rate and ODS risk to support their definition of overcorrection. It is notable that this study found the development of ODS was actually independent of the sodium correction rate. ${ }^{9}$ As for the guidelines that were cited as justification for the chosen overcorrection definitions, they too make note of the paucity of evidence guiding selection of an overcorrection definition. An evidence-based definition of hyponatremic overcorrection will require a cohort study that measures serial sodium values and ODS risk. Second, our study identified an extensive heterogeneity of overcorrection definitions which resulted in extensive variations in prevalence estimates for overcorrection that was almost 11-fold in our cohort. Therefore, without standardization amongst definitions, it is difficult to actually label a patient as overcorrected. This may have important medico-legal implications, particularly in cases where patients developed ODS. It will also complicate studies attempting to measure the association of baseline factors with overcorrection risk.

Several issues should be considered when interpreting our study. First, our systemic review did not include literature published in languages other than English. We are confident that we have included the vast majority of studies regarding the correction of hyponatremia but are uncertain how many other such studies have been conducted in other languages. However, we are certain that the essential conclusion of our study, namely that criteria for calculating correction rates and defining overcorrection are heterogeneous, would not change if studies in other languages were included in our study.

Table 6 Agreement Between Criteria Identifying Overcorrection of Patients with Severe Hyponatremia

\begin{tabular}{|c|c|c|c|c|c|c|c|c|c|c|c|c|c|c|}
\hline Criterion & A1 & B3 & B5 & C6 & D2 & E3 & E4 & E5 & F6 & G3 & G4 & H6 & I5 & I6 \\
\hline A1 & - & & & & & & & & & & & & & \\
\hline B3 & 0.176 & - & & & & & & & & & & & & \\
\hline B5 & -0.311 & 0.494 & - & & & & & & & & & & & \\
\hline C6 & -0.471 & 0.288 & 0.724 & - & & & & & & & & & & \\
\hline D2 & -0.737 & -0.048 & 0.304 & 0.388 & - & & & & & & & & & \\
\hline E3 & 0.135 & 0.792 & 0.49 & 0.317 & -0.019 & - & & & & & & & & \\
\hline E4 & -0.183 & 0.59 & 0.737 & 0.571 & 0.212 & 0.683 & - & & & & & & & \\
\hline E5 & -0.401 & 0.385 & 0.865 & 0.724 & 0.375 & 0.465 & 0.782 & - & & & & & & \\
\hline F6 & -0.449 & 0.311 & 0.747 & 0.875 & 0.381 & 0.346 & 0.619 & 0.76 & - & & & & & \\
\hline G3 & 0.218 & 0.872 & 0.404 & 0.205 & -0.119 & 0.766 & 0.51 & 0.308 & 0.24 & - & & & & \\
\hline G4 & -0.022 & 0.753 & 0.657 & 0.439 & 0.077 & 0.724 & 0.724 & 0.548 & 0.468 & 0.74 & - & & & \\
\hline H6 & -0.474 & 0.221 & 0.538 & 0.689 & 0.237 & 0.221 & 0.436 & 0.519 & 0.606 & 0.199 & 0.394 & - & & \\
\hline I5 & -0.288 & 0.516 & 0.92 & 0.702 & 0.304 & 0.538 & 0.798 & 0.83 & 0.737 & 0.426 & 0.686 & 0.519 & - & \\
\hline I6 & -0.442 & 0.33 & 0.734 & 0.946 & 0.362 & 0.353 & 0.599 & 0.721 & 0.859 & 0.253 & 0.474 & 0.74 & 0.712 & - \\
\hline
\end{tabular}

Agreement was quantified using prevalence-adjusted bias-adjusted Kappa 
Second, our cohort study was retrospective. As such, there were patients in whom insufficient data were collected to calculate the serum sodium correction rate (most notably sodium correction rate D). However, this reflects real life and would be found if the calculation rates in Table 3 were applied to other observational data. Third, while we were able to illustrate the heterogeneity in these criteria, we are unable to determine which method was best. We believe that such a determination would require a study designed to determine which of these sodium correction rate methods (Table 3) is most closely associated with ODS risk. Given the perceived low risk of this outcome, such a study would need to be very large to have the statistical power to accurately determine the association of each sodium correction rate method with ODS risk.

In conclusion, clinicians, researchers, and administrators need to be aware that extensive variations in how hyponatremia correction rates are calculated and how overcorrection is defined will have important impacts on metrics in this field. Further research is required to determine which calculation rate best predicts ODS risk.

Corresponding Author: Carl Walraven, MD; Department of MedicineThe Ottawa Hospital, ASB1-003, 1053 Carling Avenue, Ottawa, Ontario K1Y 4E9, Canada (e-mail: carlv@ohri.ca).

\section{Compliance with Ethical Standards:}

Conflict of Interest: The authors declare that they do not have a conflict of interest.

\section{REFERENCES}

1. Upadhyay A, Jaber BL, Madias NE. Incidence and Prevalence of Hyponatremia. Am J Med. 2006;119(7, Supplement 1):S30-S35. https://doi.org/10.1016/j.amjmed.2006.05.005

2. Peters M, Van der Hoeven J, Hoedemaekers C. Risk factors for poor outcome in patients with osmotic demyelination syndrome. Crit Care. 2012;16(Suppl 1):P143. https://doi.org/10.1186/cc10750

3. Lohr JW. Osmotic demyelination syndrome following correction of hyponatremia: Association with hypokalemia. Am J Med. 1994;96(5):408-413. https://doi.org/10.1016/0002-9343(94)90166-X

4. Verbalis JG, Goldsmith SR, Greenberg A, et al. Diagnosis, Evaluation, and Treatment of Hyponatremia: Expert Panel Recommendations. Am J Med. 2013;126(10):S1-S42. https://doi.org/10.1016/j.amjmed.2013.07. 006

5. Spasovski G, Vanholder R, Allolio B, et al. Clinical practice guideline on diagnosis and treatment of hyponatraemia. Eur $J$ Endocrinol. 2014;170(3):G1-G47. https://doi.org/10.1530/EJE-13-1020

6. Cheung $\mathbf{K}$, Kaur MN, Tolliver T, Longo CJ, Naam NH, Thoma A. A Tale of Two Health-Care Systems: Cost-Utility Analysis of Open Carpal Tunnel Release in Canada and the United States. Plast Surg. 2017;25(1):7-13. https://doi.org/10.1177/2292550317693817

7. Katz MA. Hyperglycemia-Induced Hyponatremia - Calculation of Expected Serum Sodium Depression. N Engl J Med. 1973;289(16):843844. https://doi.org/10.1056/NEJM197310182891607

8. Byrt T, Bishop J, Carlin JB. Bias, prevalence and kappa. J Clin Epidemiol. 1993;46(5):423-429. https://doi.org/10.1016/08954356(93)90018-V

9. Ayus JC, Krothapalli RK, Arieff AI. Treatment of Symptomatic Hyponatremia and Its Relation to Brain Damage. N Engl J Med. 1987;317(19):1190-1195. https://doi.org/10.1056/NEJM198711053171905
10. Velez JCQ, Dopson SJ, Sanders DS, Delay TA, Arthur JM. Intravenous conivaptan for the treatment of hyponatraemia caused by the syndrome of inappropriate secretion of antidiuretic hormone in hospitalized patients: a single-centre experience. Nephrol Dial Transplant. 2010;25(5): 1524-1531. https://doi.org/10.1093/ndt/gfp731

11. Jahangiri A, Wagner J, Tran MT, et al. Factors predicting postoperative hyponatremia and efficacy of hyponatremia management strategies after more than 1000 pituitary operations. J Neurosurg. 2013;119(6):14781483. https://doi.org/10.3171/2013.7.JNS13273

12. Castello LM, Baldrighi M, Panizza A, Bartoli E, Avanzi GC. Efficacy and safety of two different tolvaptan doses in the treatment of hyponatremia in the Emergency Department. Intern Emerg Med. 2017;12(7):993-1001. https://doi.org/10.1007/s11739-016-1508-5

13. Morris JH, Bohm NM, Nemecek BD, et al. Rapidity of Correction of Hyponatremia Due to Syndrome of Inappropriate Secretion of Antidiuretic Hormone Following Tolvaptan. Am J Kidney Dis. 2018;71(6):772-782. https://doi.org/10.1053/j.ajkd.2017.12.002

14. Verbalis JG, Greenberg A, Burst V, et al. Diagnosing and Treating the Syndrome of Inappropriate Antidiuretic Hormone Secretion. Am J Med. 2016;129(5):537.e9-537.e23. https://doi.org/10.1016/j.amjmed.2015. 11.005

15. Burst V, Grundmann F, Kubacki T, et al. Euvolemic hyponatremia in cancer patients. Report of the Hyponatremia Registry: an observational multicenter international study. Support Care Cancer. 2017;25(7):22752283. https://doi.org/10.1007/s00520-017-3638-3

16. Chiong JR, Hauptman PJ, Dunlap ME, Chiodo JA, Chase SL. Hospital Management of Hyponatremia in Patients with Heart Failure: Final Report from the HN Registry. J Card Fail. 2014;20(8):S62-S63. https:// doi.org/10.1016/j.cardfail.2014.06.177

17. Mohmand HK, Issa D, Ahmad Z, Cappuccio JD, Kouides RW, Sterns RH. Hypertonic Saline for Hyponatremia: Risk of Inadvertent Overcorrection. Clin J Am Soc Nephrol. 2007;2(6):1110-1117. https:// doi.org/10.2215/CJN.00910207

18. Umbrello M, Mantovani ES, Formenti $\mathbf{P}$, et al. Tolvaptan for hyponatremia with preserved sodium pool in critically ill patients. Ann Intensive Care. 2016;6. https://doi.org/10.1186/s13613-015-0096-2

19. Salahudeen AK, Ali N, George M, Lahoti A, Palla S. Tolvaptan in hospitalized cancer patients with hyponatremia: A double-blind, randomized, placebo-controlled clinical trial on efficacy and safety. Cancer. 120(5):744-751. https://doi.org/10.1002/cncr.28468

20. Schrier RW, Gross P, Gheorghiade M, et al. Tolvaptan, a Selective Oral Vasopressin V2-Receptor Antagonist, for Hyponatremia. $N$ Engl J Med. 2006;355(20):2099-2112. https://doi.org/10.1056/NEJMoa065181

21. Shoaf SE, Bricmont P, Dandurand A. Low-dose tolvaptan PK/PD: comparison of patients with hyponatremia due to syndrome of inappropriate antidiuretic hormone secretion to healthy adults. Eur $J$ Clin Pharmacol. 2017;73(11):1399-1408. https://doi.org/10.1007/s00228017-2302-7

22. George JC, Zafar W, Bucaloiu ID, Chang AR. Risk Factors and Outcomes of Rapid Correction of Severe Hyponatremia. Clin J Am Soc Nephrol CJASN. June 2018. https://doi.org/10.2215/CJN. 13061117

23. Soupart A, Gross P, Legros J-J, et al. Successful Long-Term Treatment of Hyponatremia in Syndrome of Inappropriate Antidiuretic Hormone Secretion with Satavaptan (SR121463B), an Orally Active Nonpeptide Vasopressin V2-Receptor Antagonist. Clin J Am Soc Nephrol. 2006; 1(6):1154-1160. https://doi.org/10.2215/CJN.00160106

24. Kim G, Heo B, Oh E, et al. Management of Recipients With Hyponatremia During and After Liver Transplantation; Ten Year Experience of Single Center.: Abstract\# D2628. Transplantation. 2014;98:791.

25. Romanovsky A, Azevedo LCP, Meeberg G, Zibdawi R, Bigam D, Bagshaw SM. Serum sodium shift in hyponatremic patients undergoing liver transplantation: a retrospective cohort study. Ren Fail. 2015;37(1):37-44. https://doi.org/10.3109/0886022X.2014.975102

26. Crook MA, Velauthar U, Moran L, Griffiths W. Review of Investigation and Management of Severe Hyponatremia in a Hospital Population. Ann Clin Biochem. 1999;36(2):158-162. https://doi.org/10.1177/ 000456329903600204

27. Sood L, Sterns RH, Hix JK, Silver SM, Chen L. Hypertonic Saline and Desmopressin: A Simple Strategy for Safe Correction of Severe Hyponatremia. Am J Kidney Dis. 2013;61(4):571-578. https://doi.org/ 10.1053/j.ajkd.2012.11.032

28. Winzeler B, Jeanloz $\mathbf{N}$, Nigro $\mathbf{N}$, et al. Long-term outcome of profound hyponatremia: a prospective 12 months follow-up study. Eur $J$ Endocrinol. 2016;175(6):499-507. https://doi.org/10.1530/EJE-160500 
29. MacMillan TE, Cavalcanti RB. Outcomes in Severe Hyponatremia Treated With and Without Desmopressin. Am J Med. 2018;131(3):317.e1-317.e10. https://doi.org/10.1016/j.amjmed.2017. 09.048

30. Burst V, Grundmann F, Kubacki T, et al. Thiazide-Associated Hyponatremia, Report of the Hyponatremia Registry: An Observational Multicenter International Study. Am J Nephrol. 2017;45(5):420-430. https://doi.org/10.1159/000471493

31. Rafat C, Schortgen F, Gaudry S, et al. Use of desmopressin acetate in severe hyponatremia in the intensive care unit. Clin J Am Soc Nephrol CJASN. 2014;9(2):229-237. https://doi.org/10.2215/CJN.00950113

32. Ward FL, Tobe Sw, Naimark DMJ. The Role of Desmopressin in the Management of Severe, Hypovolemic Hyponatremia: A Single-Center, Comparative Analysis. Can J Kidney Health Dis. 2018;5. https://doi.org/ $10.1177 / 2054358118761051$
33. Ghali JK, Koren MJ, Taylor JR, et al. Efficacy and Safety of Oral Conivaptan: A V1A/V2 Vasopressin Receptor Antagonist, Assessed in a Randomized, Placebo-Controlled Trial in Patients with Euvolemic or Hypervolemic Hyponatremia. J Clin Endocrinol Metab. 2006;91(6):21452152. https://doi.org/10.1210/jc.2005-2287

34. Abraham WT, Hensen J, Gross PA, et al. Lixivaptan safely and effectively corrects serum sodium concentrations in hospitalized patients with euvolemic hyponatremia. Kidney Int. 2012;82(11):1223-1230. https:// doi.org/10.1038/ki.2012.275

Publisher's Note Springer Nature remains neutral with regard to jurisdictional claims in published maps and institutional affiliations. 